\title{
Automata and Formal Languages
}

\author{
Peter Wood
}

Department of Computer Science and Information Systems

Birkbeck, University of London

ptw@dcs.bbk.ac.uk 


\section{Outline}

Peter Wood

Motivation and Background

Automata

Grammars

Regular Expressions

Example of Research

Conclusion 


\section{Doing Research}

- analysing problems/languages

- computability/solvability/decidability — is there an algorithm?

- computational complexity - is it practical?

- expressive power - are there things that cannot be expressed?

- formal languages provide well-studied models 


\section{Formal Languages}

- given a finite alphabet (set) of symbols $\Sigma$

- e.g., $\Sigma=\{0,1\}$

- a string is a sequence (concatenation) of symbols

- e.g., 0101

- all finite strings over $\Sigma$ are denoted by $\Sigma^{*}$

- e.g., $\Sigma^{*}=\{\epsilon, 0,1,00,01,10,11, \ldots\}$

- language $L$ over $\Sigma$ is just a subset of $\Sigma^{*}$

- e.g., $L_{1}$ : strings with an even number of 1 's

- e.g., $L_{0}$ : strings representing valid Java programs (over an alphabet of all legal symbols in Java)

- are there finite representations for infinite languages? 


\section{Formal Languages}

- given a finite alphabet (set) of symbols $\Sigma$

- e.g., $\Sigma=\{0,1\}$

- a string is a sequence (concatenation) of symbols

- e.g., 0101

- all finite strings over $\Sigma$ are denoted by $\Sigma^{*}$

- e.g., $\Sigma^{*}=\{\epsilon, 0,1,00,01,10,11, \ldots\}$

- language $L$ over $\Sigma$ is just a subset of $\Sigma^{*}$

- e.g., $L_{1}$ : strings with an even number of 1 's

- e.g., $L_{0}$ : strings representing valid Java programs (over an alphabet of all legal symbols in Java)

- are there finite representations for infinite languages?

- yes, grammars (generative) and automata (recognition) 


\section{Automata}

- device (machine) for recognising (accepting) a language

- provide models of computation

- automaton comprises states and transitions between states

- automaton is given a string as input

- automaton $M$ accepts a string $w$ by halting in an accept state, when given $w$ as input

- language $L(M)$ accepted by automaton $M$ is the set of all strings which $M$ accepts 


\section{Types of Automata}

- finite state automaton

- deterministic

- nondeterministic

- pushdown automaton

- linear-bounded automaton

- Turing machine 


\section{Example of a Finite State Automaton}

- $L_{1}$ (strings with an even number of 1's) can be recognised by the following FSA

- 2 states $s_{\text {even }}$ and $s_{\text {odd }}$

- 4 transitions

- $s_{\text {even }}$ is both the initial and final state

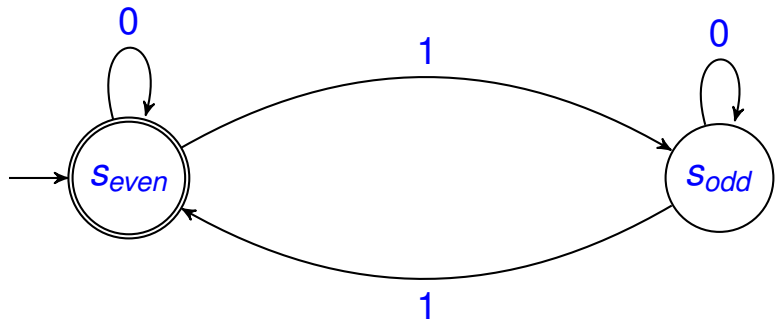

\section{Automata}

- FSA recognises 011: 


\section{Example of a Finite State Automaton}

- $L_{1}$ (strings with an even number of 1's) can be recognised by the following FSA

- 2 states $s_{\text {even }}$ and $s_{\text {odd }}$

- 4 transitions

- $s_{\text {even }}$ is both the initial and final state

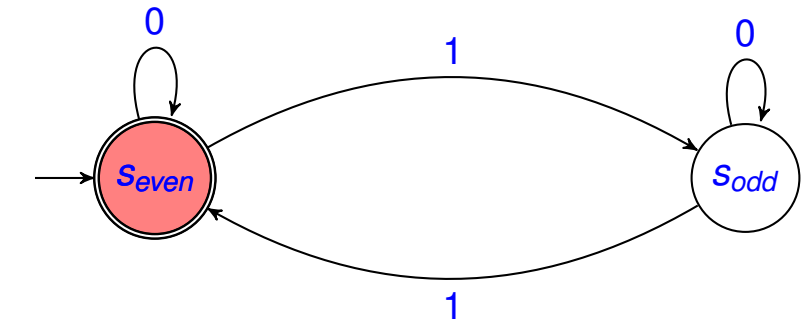

\section{Automata}

- FSA recognises 011: 


\section{Example of a Finite State Automaton}

- $L_{1}$ (strings with an even number of 1's) can be recognised by the following FSA

- 2 states $s_{\text {even }}$ and $s_{\text {odd }}$

- 4 transitions

- $s_{\text {even }}$ is both the initial and final state

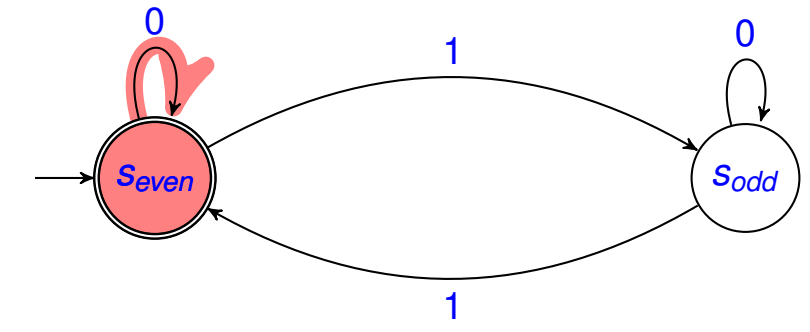

\section{Automata}

- FSA recognises 011: 0 


\section{Example of a Finite State Automaton}

- $L_{1}$ (strings with an even number of 1's) can be recognised by the following FSA

- 2 states $s_{\text {even }}$ and $s_{\text {odd }}$

- 4 transitions

- $s_{\text {even }}$ is both the initial and final state

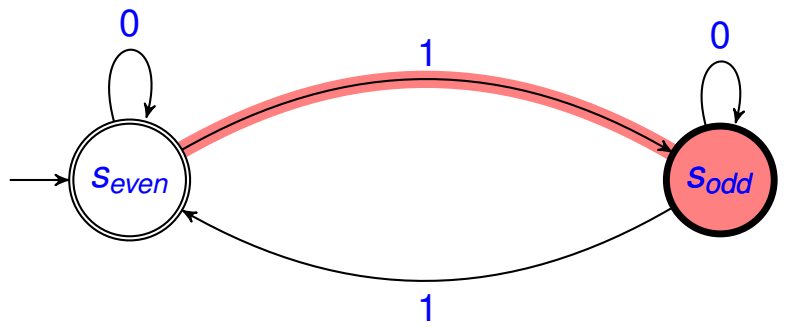

\section{Automata}

Peter Wood

Motivation and

Background

Grammars

Regular

Expressions

Example of

Research

Conclusion

- FSA recognises 011: 01 


\section{Example of a Finite State Automaton}

- $L_{1}$ (strings with an even number of 1's) can be recognised by the following FSA

- 2 states $s_{\text {even }}$ and $s_{\text {odd }}$

- 4 transitions

- $s_{\text {even }}$ is both the initial and final state

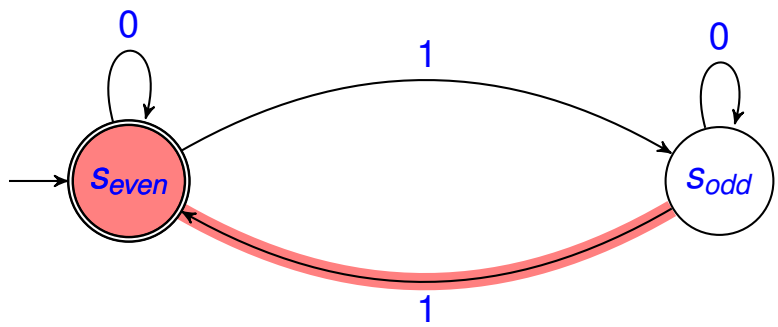

\section{Automata}

Peter Wood

Motivation and

Background

Grammars

Regular

Expressions

Example of

Research

Conclusion

- FSA recognises 011: 011 


\section{Grammars}

- grammars generate languages using:

- symbols from alphabet $\Sigma$ (called terminals)

- set $N$ of nonterminals (one designated as starting)

- set $P$ of productions, each of the form

$$
U \rightarrow V
$$

where $U$ and $V$ are (loosely) strings over $\Sigma \cup N$

- a string (sequence of terminals) $w$ is generated by $G$ if there is a derivation of $w$ using $G$, starting from the starting nonterminal of $G$

- language generated by grammar $G$, denoted $L(G)$, is the set of strings which can be derived using $G$ 


\section{Grammar Example}

- $L_{1}$ (strings with an even number of 1's) can be generated by a grammar with productions

$$
\begin{aligned}
& S \rightarrow \epsilon \\
& S \rightarrow 0 S \\
& S \rightarrow 1 T \\
& T \rightarrow 0 T \\
& T \rightarrow 1 S
\end{aligned}
$$

where $S$ is the starting nonterminal 


\section{Grammar Example}

- $L_{1}$ (strings with an even number of 1's) can be generated by a grammar with productions

$$
\begin{aligned}
& S \rightarrow \epsilon \\
& S \rightarrow 0 S \\
& S \rightarrow 1 T \\
& T \rightarrow 0 T \\
& T \rightarrow 1 S
\end{aligned}
$$

where $S$ is the starting nonterminal

- a derivation of 01010 is given by

$$
S \Rightarrow 0 \underline{S}
$$

Automata and Formal Languages

Peter Wood 


\section{Grammar Example}

- $L_{1}$ (strings with an even number of 1's) can be generated by a grammar with productions

$$
\begin{aligned}
& S \rightarrow \epsilon \\
& S \rightarrow 0 S \\
& S \rightarrow 1 T \\
& T \rightarrow 0 T \\
& T \rightarrow 1 S
\end{aligned}
$$

where $S$ is the starting nonterminal

- a derivation of 01010 is given by

$$
S \Rightarrow 0 \underline{S} \Rightarrow 01 \underline{I}
$$




\section{Grammar Example}

- $L_{1}$ (strings with an even number of 1's) can be generated by a grammar with productions

$$
\begin{aligned}
& S \rightarrow \epsilon \\
& S \rightarrow 0 S \\
& S \rightarrow 1 T \\
& T \rightarrow 0 T \\
& T \rightarrow 1 S
\end{aligned}
$$

where $S$ is the starting nonterminal

- a derivation of 01010 is given by

$$
S \Rightarrow 0 \underline{S} \Rightarrow 01 \underline{T} \Rightarrow 010 \underline{I}
$$

Automata and Formal Languages

Peter Wood

Grammars

Regular

Expressions

Example of

Research

Conclusion 


\section{Grammar Example}

- $L_{1}$ (strings with an even number of 1's) can be generated by a grammar with productions

$$
\begin{aligned}
& S \rightarrow \epsilon \\
& S \rightarrow 0 S \\
& S \rightarrow 1 T \\
& T \rightarrow 0 T \\
& T \rightarrow 1 S
\end{aligned}
$$

where $S$ is the starting nonterminal

- a derivation of 01010 is given by

$$
S \Rightarrow 0 \underline{S} \Rightarrow 01 \underline{I} \Rightarrow 010 \underline{T} \Rightarrow 0101 \underline{S}
$$




\section{Grammar Example}

- $L_{1}$ (strings with an even number of 1's) can be generated by a grammar with productions

$$
\begin{aligned}
& S \rightarrow \epsilon \\
& S \rightarrow 0 S \\
& S \rightarrow 1 T \\
& T \rightarrow 0 T \\
& T \rightarrow 1 S
\end{aligned}
$$

where $S$ is the starting nonterminal

- a derivation of 01010 is given by

$$
S \Rightarrow 0 \underline{S} \Rightarrow 01 \underline{I} \Rightarrow 010 \underline{I} \Rightarrow 0101 \underline{S} \Rightarrow 01010 \underline{S}
$$

Automata and Formal Languages

Peter Wood

Grammars

Regular

Expressions

Example of

Research

Conclusion 


\section{Grammar Example}

- $L_{1}$ (strings with an even number of 1's) can be generated by a grammar with productions

$$
\begin{aligned}
& S \rightarrow \epsilon \\
& S \rightarrow 0 S \\
& S \rightarrow 1 T \\
& T \rightarrow 0 T \\
& T \rightarrow 1 S
\end{aligned}
$$

where $S$ is the starting nonterminal

- a derivation of 01010 is given by

$$
S \Rightarrow 0 \underline{S} \Rightarrow 01 \underline{I} \Rightarrow 010 \underline{I} \Rightarrow 0101 \underline{S} \Rightarrow 01010 \underline{S} \Rightarrow 01010
$$




\section{Uses of Grammars}

- to specify syntax of programming languages

- in natural language understanding

- in pattern recognition

- to specify schemas (types) for tree-structured data, e.g., XML 


\section{Hierarchy of Grammars and Languages}

- restrictions on productions give different types of grammars

- regular (type 3)

- context-free (type 2)

- context-sensitive (type 1)

- phrase-structure (type 0)

- for context-free, e.g., left side must be single nonterminal

- no restrictions for phrase-structure

- language is of type $i$ iff there is a grammar of type $i$ which generates it 


\section{Examples of Language Hierarchy}

Peter Wood

- varying expressive power

- regular $\subset$ context-free $\subset$ context-sensitive $\subset$ phrase-structure 


\section{Examples of Language Hierarchy}

- varying expressive power

- regular $\subset$ context-free $\subset$ context-sensitive $\subset$ phrase-structure

- $L_{1}$ (strings over $\{0,1\}$ with an even number of 1 's) is regular 


\section{Examples of Language Hierarchy}

- varying expressive power

- regular $\subset$ context-free $\subset$ context-sensitive $\subset$ phrase-structure

- $L_{1}$ (strings over $\{0,1\}$ with an even number of 1 's) is regular

- $L_{2}=\left\{0^{n} 1^{n} \mid n \geq 0\right\}$ is context-free, but not regular 


\section{Examples of Language Hierarchy}

- varying expressive power

- regular $\subset$ context-free $\subset$ context-sensitive $\subset$ phrase-structure

- $L_{1}$ (strings over $\{0,1\}$ with an even number of 1 's) is regular

- $L_{2}=\left\{0^{n} 1^{n} \mid n \geq 0\right\}$ is context-free, but not regular

- $L_{3}=\left\{w w \mid w \in\{0,1\}^{*}\right\}$ is context-sensitive, but not context-free 


\section{Examples of Language Hierarchy}

- varying expressive power

- regular $\subset$ context-free $\subset$ context-sensitive $\subset$ phrase-structure

- $L_{1}$ (strings over $\{0,1\}$ with an even number of 1 's) is regular

- $L_{2}=\left\{0^{n} 1^{n} \mid n \geq 0\right\}$ is context-free, but not regular

- $L_{3}=\left\{w w \mid w \in\{0,1\}^{*}\right\}$ is context-sensitive, but not context-free

- there exists a phrase-structure (recursive) language which is not context-sensitive 


\section{Complexity of Grammar Problems}

Peter Wood

\begin{tabular}{lcccc}
\hline \multicolumn{1}{c}{ Problem } & \multicolumn{4}{c}{ Type } \\
\cline { 2 - 5 } & 3 & 2 & 1 & 0 \\
\hline Is $w \in L(G) ?$ & $\mathrm{P}$ & $\mathrm{P}$ & PSPACE & $\mathrm{U}$ \\
Is $L(G)$ empty? & $\mathrm{P}$ & $\mathrm{P}$ & $\mathrm{U}$ & $\mathrm{U}$ \\
Is $L\left(G_{1}\right) \equiv L\left(G_{2}\right) ?$ & PSPACE & $\mathrm{U}$ & $\mathrm{U}$ & $\mathrm{U}$ \\
\hline
\end{tabular}

- P: decidable in polynomial time

- PSPACE: decidable in polynomial space (and complete for PSPACE: at least as hard as NP-complete)

- U: undecidable

- so type of grammar has significant effect on complexity 


\section{Relationships between Languages and Automata}

A language is

$\left.\begin{array}{c}\text { regular } \\ \text { context-free } \\ \text { context-sensitive } \\ \text { phrase-structure }\end{array}\right\} \begin{gathered}\text { iff } \\ \text { accepted } \\ \text { by }\end{gathered} \quad\left\{\begin{array}{c}\text { finite-state } \\ \text { pushdown } \\ \text { linear-bounded } \\ \text { Turing machine }\end{array}\right.$




\section{Regular Expressions}

- algebraic notation for denoting regular languages

- use $\circ$ (concatenation), $\cup$ (union) and $*$ (closure) operators

- $L_{1}$ denoted by $\mathrm{RE} 0^{*} \cup\left(0^{*} \circ 1 \circ 0^{*} \circ 1 \circ 0^{*}\right)^{*}$

- given RE $R$, the set of strings it denotes is $L(R)$

- pattern matching in text

- query languages for XML or RDF 
Graphs (networks) are widely used for representing data

- social networks

- transportation and other networks

- geographical information

- semistructured data

- (hyper)document structure

- semantic associations in criminal investigations

- bibliographic citation analysis

- pathways in biological processes

- knowledge representation (e.g. semantic web)

- program analysis

- workflow systems

- data provenance 
- (my PhD thesis!)

- usually regular expressions used for string search

- consider data represented by a directed graph of labelled nodes and labelled edges

- regular expressions can express paths we are interested in

- sequence of edge labels rather than sequence of symbols (characters)

- a query using regular expression $R$ can ask for all nodes connected by a path whose concatenation of edge labels is in $L(R)$ 
Graph $G$ (where nodes represent people and places):

Peter Wood

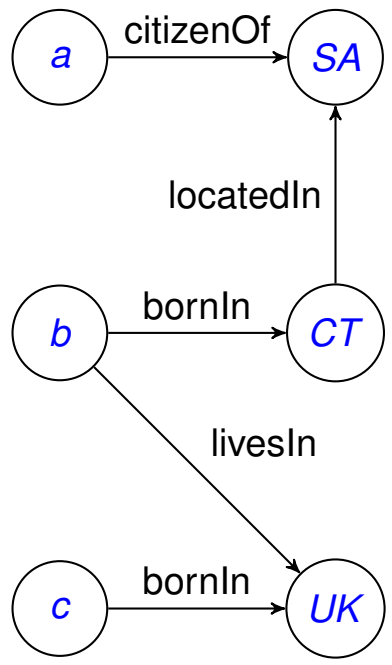

Motivation and

Background

Automata

Grammars

Regular:

Expressions

Example of

Research 
Regular expression

$R=$ citizenOf $\mid$ ((bornln | livesIn) · locatedln*)

asks for paths of edges between a person $x$ and a place $y$ such that

- $x$ is a citizenOf $y$, or

- $x$ is bornln or livesln $y$, or

- $x$ is bornln or livesIn a place that is locatedln $y$ 


\section{Regular path query evaluation}

- Regular Path Problem

Given graph $G$, pair of nodes $x$ and $y$ and regular expression $R$, is there a path from $x$ to $y$ satisfying $R ?$

- algorithm:

- construct a nondeterministic finite automaton (NFA) $M$ accepting $L(R)$

- assume $M$ has initial state $s_{0}$ and final state $s_{f}$

- consider $G$ as an NFA with initial state $x$ and final state $y$

- form the "intersection" (or "product") $I$ of $M$ and $G$

- check if there is a path from $\left(s_{0}, x\right)$ to $\left(s_{f}, y\right)$

- Each step can be done in PTIME, so REgulAR PATH PROBLEM has PTIME complexity 
NFA $M$ for $R=$ citizenOf $\mid(($ bornln $\mid$ livesln $) \cdot$ locatedln*)

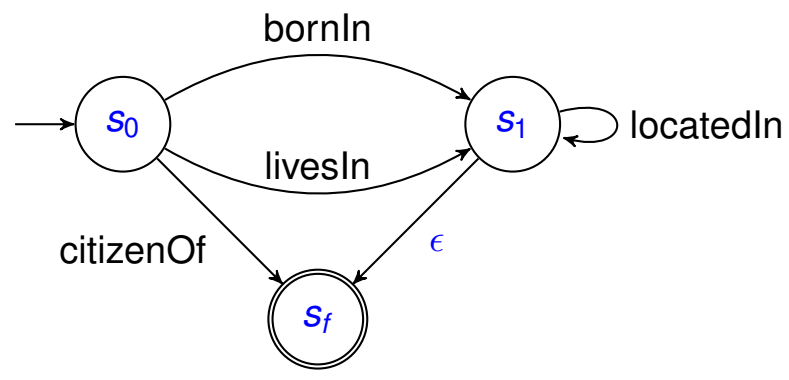

\section{Example of \\ Research}


Peter Wood

citizenOf

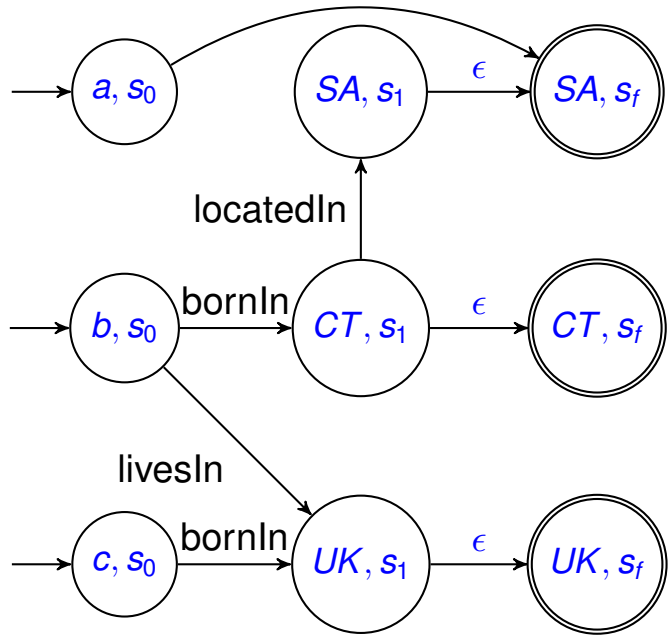




\section{citizenOf}

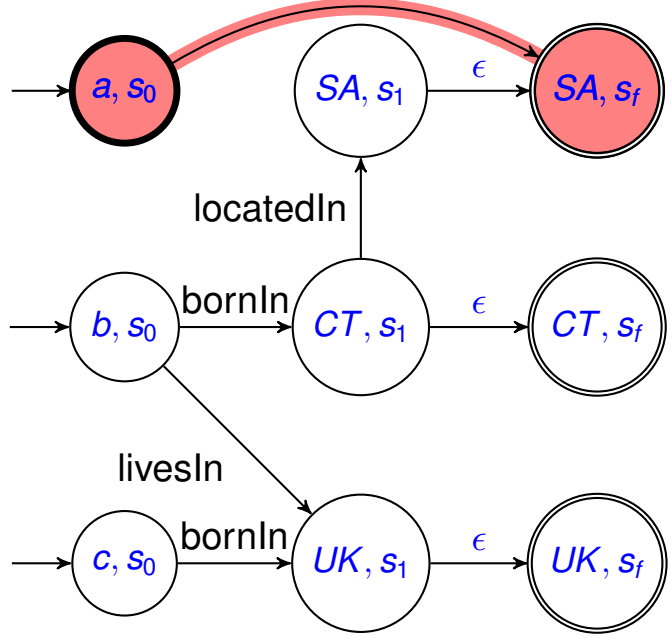

\section{Example of \\ Research}


Peter Wood

\section{citizenOf}
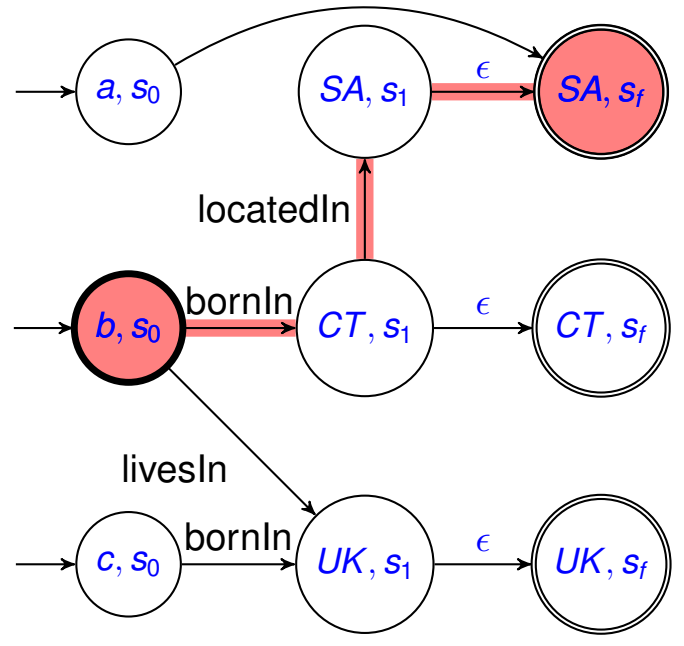

\section{Example of \\ Research}

\section{Conclusion}


Peter Wood

citizenOf

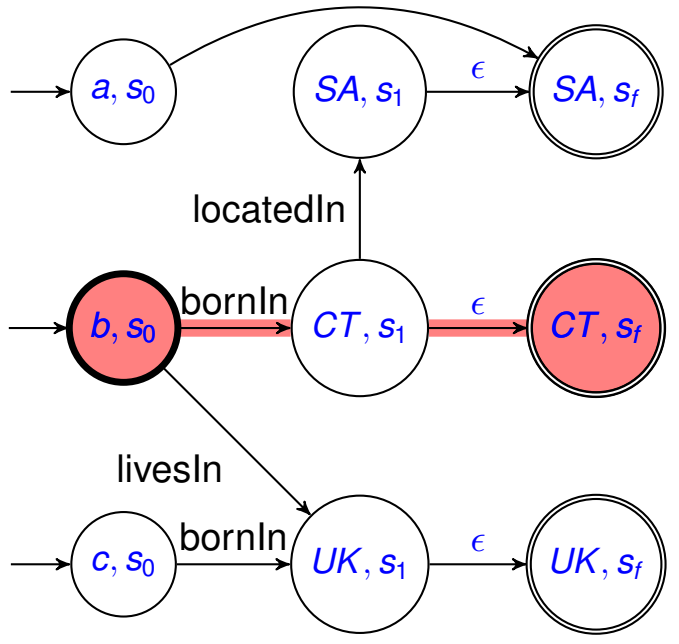


Peter Wood

\section{citizenOf}

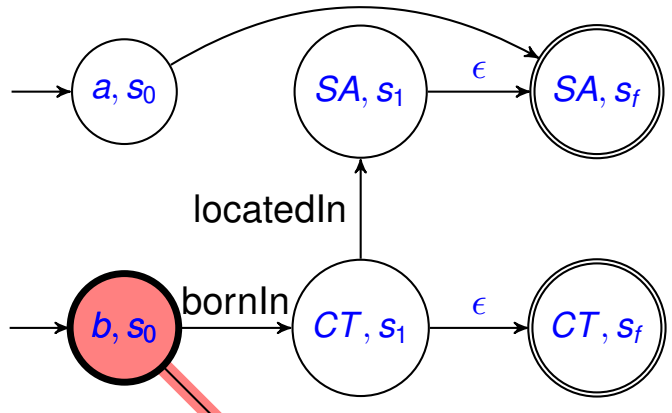

Example of

Research

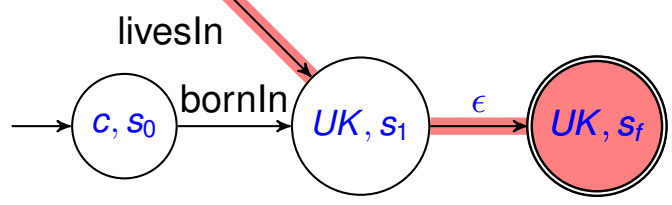


Peter Wood

\section{citizenOf}

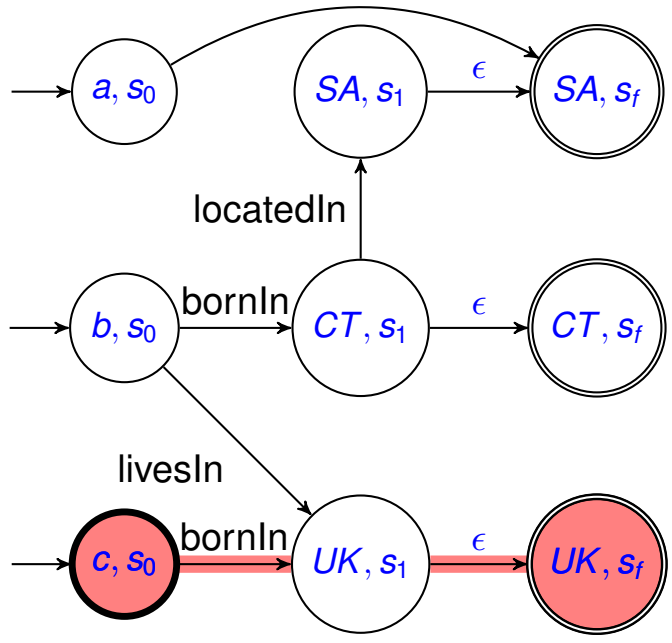

\section{Example of \\ Research}




\section{Regular simple path queries}

- path $p$ is simple if no node is repeated on $p$

- Regular Simple Path Problem

Given graph $G$, pair of nodes $x$ and $y$ and regular expression $R$, is there a simple path from $x$ to $y$ satisfying $R$ ? 


\section{Regular simple path queries}

- path $p$ is simple if no node is repeated on $p$

- Regular Simple Path Problem Given graph $G$, pair of nodes $x$ and $y$ and regular expression $R$, is there a simple path from $x$ to $y$ satisfying $R$ ?

- Regular Simple Path Problem is NP-complete [Mendelzon \& Wood (1989)] 


\section{Regular simple path queries}

Peter Wood

- path $p$ is simple if no node is repeated on $p$

- Regular Simple Path Problem Given graph $G$, pair of nodes $x$ and $y$ and regular expression $R$, is there a simple path from $x$ to $y$ satisfying $R$ ?

- Regular Simple Path Problem is NP-complete [Mendelzon \& Wood (1989)]

- there can be a path from $x$ to $y$ satisfying $R$ but no simple path satisfying $R$, e.g., $R=(c \cdot d)^{*}$

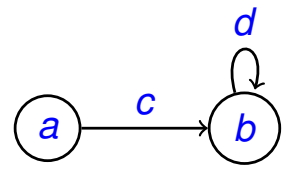




\section{Approaches to deal with this problem}

- what causes the problem? 


\section{Approaches to deal with this problem}

- what causes the problem?

- the presence of cycles 


\section{Approaches to deal with this problem}

- what causes the problem?

- the presence of cycles

- obvious first step is to consider graphs without cycles-DAGs 


\section{Approaches to deal with this problem}

- what causes the problem?

- the presence of cycles

- obvious first step is to consider graphs without cycles-DAGs

- then might look at restricted forms of REs-we looked at those corresponding to languages closed under abbreviations 


\section{Approaches to deal with this problem}

- what causes the problem?

- the presence of cycles

- obvious first step is to consider graphs without cycles-DAGs

- then might look at restricted forms of REs-we looked at those corresponding to languages closed under abbreviations

- then one might consider a combination of graphs and REs-we looked at graphs whose cycle structure does not conflict with the RE 


\section{Approaches to deal with this problem}

- what causes the problem?

Peter Wood

- the presence of cycles

- obvious first step is to consider graphs without cycles-DAGs

- then might look at restricted forms of REs-we looked at those corresponding to languages closed under abbreviations

- then one might consider a combination of graphs and REs-we looked at graphs whose cycle structure does not conflict with the RE

- finally showed that conflict-freedom is a generalisation:

- no RE conflicts with any DAG

- an RE closed under abbreviations never conflicts with any graph 


\section{Other approaches}

Automata and

Peter Wood

- in general, may also run experiments to measure actual running times

Motivation and

Background

Automata

Grammars

Regular

Expressions

Example of

Research

Conclusion 


\section{Other approaches}

- in general, may also run experiments to measure actual running times

- may also develop approximation algorithms

- can sometimes find a PTIME algorithm with a performance guarantee (e.g. for TSP, finds a tour at most twice the optimal distance)

- other times this problem itself is NP-hard 


\section{Conclusion}

- is my system/language more powerful than others?

- is my system/language more efficient than others?

- expressive power or computational complexity can be studied by relating them to

- formal language theory: languages, grammars, automata, ...

- tradeoff between expressive power and computational complexity

- consider restrictions of difficult problems or giving up exact solutions 


\section{References}

- Aho, Hopcroft and Ullman, "The Design and Analysis of Computer Algorithms," Addison-Wesley, 1974

- Garey and Johnson, "Computers and Intractability: $A$ Guide to the Theory of NP-Completeness," W. H. Freeman and Company, 1979

- Lewis and Papadimitriou, "Elements of the Theory of Computation," Prentice-Hall, 1981

- Mendelzon and Wood, "Finding Regular Simple Paths in Graph Databases," SIAM J. Computing, Vol. 24. No. 6, 1995, pp. 1235-1258

- Sipser, "Introduction to the Theory of Computation," PWS Publishing Company, 1997 